\title{
Spectral Features of KT Eri in its Nebular Phase
}

\author{
Akira Arai ${ }^{1}$, Mizuki Isoggai $^{1}$, Kazuyoshi Imamura $^{2}$, Yuji Ikeda $^{3}$, \\ Takayuki Arasaki ${ }^{3}$, Eiji Kitao ${ }^{3}$, and Gaku Taguchi ${ }^{3}$ \\ ${ }^{1}$ Koyama Astronomical Observatory, Kyoto Sangyo University, Motoyama, Kamigamo, \\ Kita-ku, Kyoto, Japan, 603-8555 \\ ${ }^{2}$ Department of Biosphere-Geosphere System Science, Faculty of Informatics, Okayama \\ University of Science, 1-1 Ridai-cho, Okayama, Okayama, Japan, 700-0005 \\ ${ }^{3}$ Department of Physics, Kyoto Sangyo University, Motoyama, Kamigamo, Kita-ku, Kyoto, \\ Japan, 603-8555 \\ email: arai6a@cc.kyoto-su.ac.jp
}

\begin{abstract}
Non-spherically symmetric distributions of ejected gas are often observed in classical nova remnants. However, it is unclear if these asymmetries could be produced from nova explosions. Spectra in the nebular phase can provide information on the kinematics of ejected materials. Classical nova KT Eri was discovered on November 2009. It is considered a candidate for a recurrent nova based on the high expansion velocity and rapid decline of its light curve. In order to investigate the velocity field of ejected gas in the system, we performed optical spectroscopic observations from 2010 September to 2010 December at Koyama Astronomical Observatory. We noticed the strong [O III] and He II lines in the spectra on September 1, which suggests that the nova had already entered the nebular phase. It is notable that the [O III] lines showed complex profiles with at least six peaks on September 1. Those features can be de-convolved into four velocity components $\left(-2,000 \mathrm{~km} \mathrm{~s}^{-1},-1,000 \mathrm{~km} \mathrm{~s}^{-1},+700 \mathrm{~km} \mathrm{~s}^{-1}\right.$, and $\left.+1,800 \mathrm{~km} \mathrm{~s}^{-1}\right)$ of doublet [O III] $\lambda 4959$ and $\lambda 5007$.
\end{abstract}

Keywords. stars: novae, cataclysmic variables - techniques: spectroscopic

In many classical novae, non-spherically symmetric distributions of ejected gas are observed. However, it is unclear if these asymmetries can be produced from nova explosions. Morphological studies of several novae have been recently presented (e.g., V2491 Cyg, Ribeiro et al. 2011; and V2672 Oph, Munari et al. 2011a). Spectra in the nebular phase also reveal hints of velocity fields of optically thin gas, and it is important to compare with those in the early phase. Here, we report on low dispersion spectroscopic observations of a very fast nova, KT Eri, in its nebular phase. KT Eri was discovered on 2009 November 25.536UT at $8.1 \mathrm{mag}$ by K. Itagaki (Yamaoka et al. 2009) 13 days after the maximum. SMEI observed around the maximum on 2009 November 14.67UT (Hounsell et al. 2010). Based on the $t_{2} V \sim 6.6 \mathrm{~d}$ (Hounsell et al. 2010), the nova was classified as a very fast nova. Its spectral type was He/N type nova, and the FWHM was 3,000 km $\mathrm{s}^{-1}$ for the $\mathrm{H}_{\alpha}$ emission (Yamaoka et al. 2009).

Our observations were performed from 2010 September 01 to 2010 December 26 using the $1.3 \mathrm{~m}$ Araki telescope with LOSA/F2 (Low-dispersion Optical Spectrograph for Araki telescope with F/2 optics) at Koyama Astronomical Observatory, Kyoto Sangyo University, Japan.

Figure 1 shows the spectrum on 2010 September 01, 291 days after the maximum. The spectrum exhibits $\mathrm{H} \alpha, \mathrm{H} \beta$, He II 4686 and broad six-peaked [O III] emission, which indicates that KT Eri was in its nebular phase because fluxes of He II and [O III] are stronger than any other emission lines. We noticed that doublet [O III] $\lambda 4959$ and $\lambda 5007$ shows 


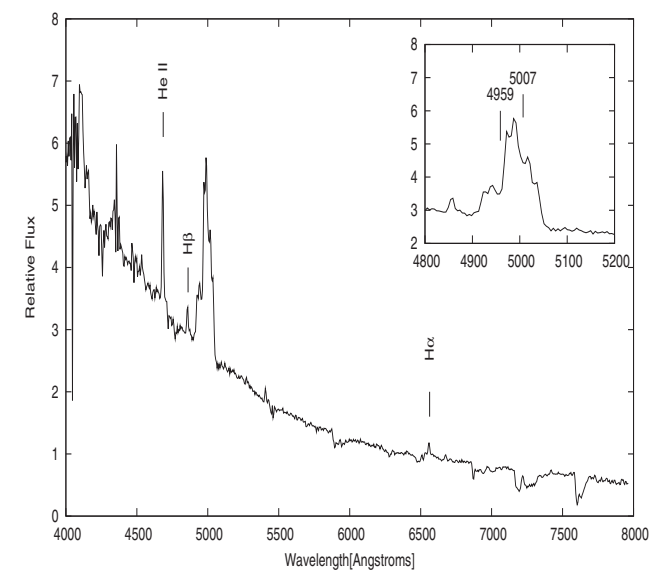

Figure 1. A nebular phase spectrum of KT Eri on September 1, 2011.

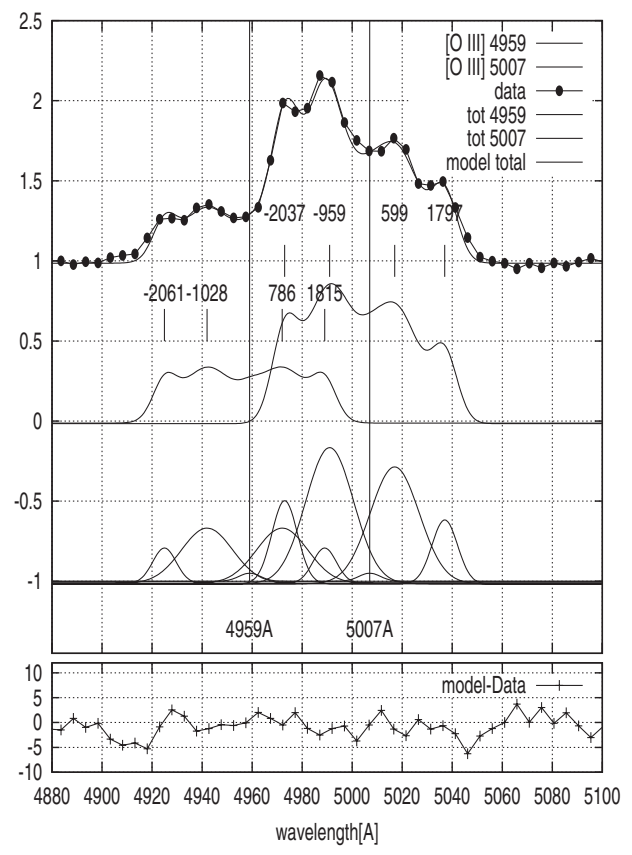

Figure 2. De-convolved spectra of [O III] emission lines.

a complex profile and it was similar to those of previous novae in their nebular phase (e.g., V444 Sct, V4160 Sgr and V2491 Cyg; Williams et al. 1994, Munari et al. 2011b). Figure 2 shows de-convolved [O III] profiles on September 1 fitted by the 10 Gaussian components. Two center components are put at rest wavelengths for each [O III] center. The other four Gaussian components for each [O III] emission are centered at -2,100, $1,000,+800$, and $+1,800 \mathrm{~km} \mathrm{~s}^{-1}$. These velocity components are consistent with doublet [O III]. Calculations of emission line profiles of novae are presented in Gill and O'Brien (1999). They reproduced emission-line profiles from the model of nova shells with various symmetries. We suggest that the observed and de-convolved shape of [O III] lines would correspond to "two rings" or "polar caps with an equatorial ring" seen most probably near 60 deg from edge-on, by comparing with their results. These results suggest that the existence of expanding ring structures with observed line separations of $2,800 \mathrm{~km} \mathrm{~s}^{-1}$.

\section{References}

Gill, C. D. \& O'Brien, T. J. 1999, MNRAS 307, 677

Hounsell, R., Bode, M. F., Hick, P. P., Buffington, A., Jackson, B. V., Clover, J. M., Shafter, A. W., Darnley, M. J., Mawson, N. R., Steele, I. A., Evans, A., Eyres, S. P. S., \& O’Brien, T. J. 2010, ApJ 724, 480

Munari, U., Ribeiro, V. A. R. M., Bode, M. F., \& Saguner, T. 2011, MNRAS 410, 525

Munari, U., Siviero, A., Dallaporta, S., Cherini, G., Valisa, P., \& Tomasella, L. 2011, NewAstron. 16,209

Ribeiro, V. A. R. M., Darnley, M. J., Bode, M. F., Munari, U., Harman, D. J., Steele, I. A., \& Meaburn, J. 2011, MNRAS 412, 1701

Yamaoka, H., Itagaki, K., Guido, E., Maehara, H., Fujii, M., Arai, A., Isogai, M., Immura, K., Rudy, R. J., Prater, T. R., Russell, R. W., Puetter, R. C., Perry, R. B., \& Kazarovets, E. V. 2009, IAU Circ. 9098, 1

Williams, R. E., Phillips, M. M., \& Hamuy, M. 1994, ApJS 90, 297 